\title{
Michel Beaud, Face au pire des mondes, Paris, Seuil, 2011, 293 p.
}

Igor Martinache

\section{(2) OpenEdition}

Journals

Édition électronique

URL : http://journals.openedition.org/developpementdurable/9193

DOI : 10.4000/developpementdurable.9193

ISSN : 1772-9971

Éditeur

Association DD\&T

Référence électronique

Igor Martinache, « Michel Beaud, Face au pire des mondes, Paris, Seuil, 2011, 293 p. », Développement durable et territoires [En ligne], Vol. 3, nº 1 | Mai 2012, mis en ligne le 21 mai 2012, consulté le 22 septembre 2020. URL : http://journals.openedition.org/developpementdurable/9193 ; DOI : https:// doi.org/10.4000/developpementdurable.9193

Ce document a été généré automatiquement le 22 septembre 2020.

Développement Durable et Territoires est mis à disposition selon les termes de la licence Creative Commons Attribution - Pas d'Utilisation Commerciale 4.0 International. 


\title{
Michel Beaud, Face au pire des mondes, Paris, Seuil, 2011, 293 p.
}

\author{
Igor Martinache
}

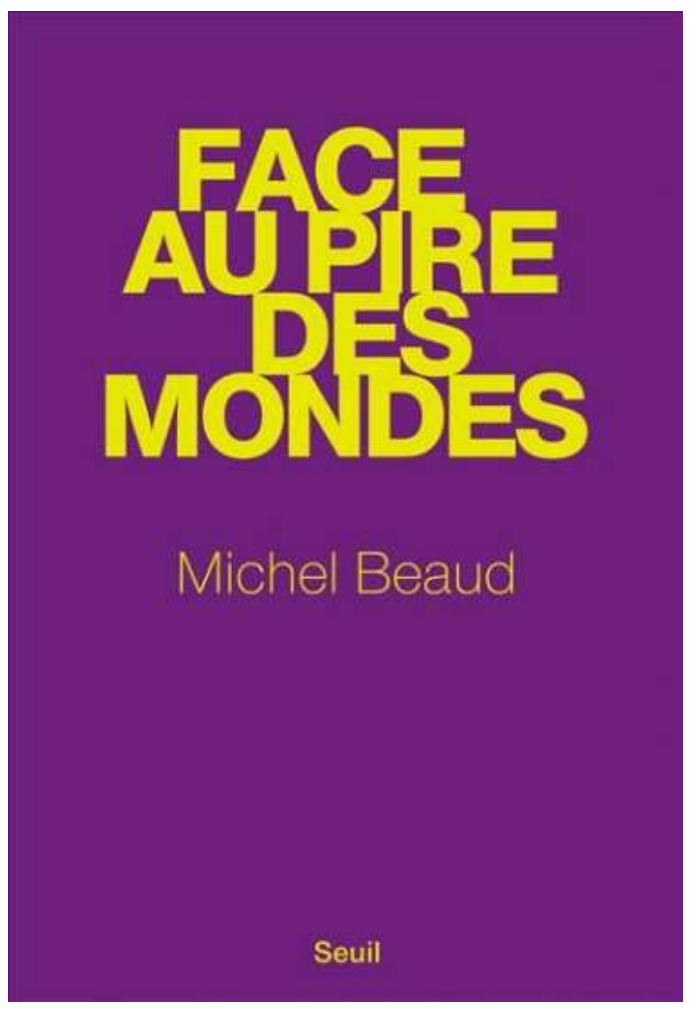

1 Parmi les auteurs d'essai, il en est qui, au-delà des « faits » et analyses présentés, savent adopter un ton personnel pour mieux saisir leurs lecteurs. Michel Beaud est sans conteste de ceux-là, qui, en dépit d'une impressionnante carrière universitaire, savent s'affranchir des carcans académiques pour lancer un appel à la raison économique, en ne s'embarrassant pas de pronoms“impersonnels, de périphrases «politiquement correctes ", et en s'appuyant essentiellement sur des informations tirées d'Internet, notamment du site du quotidien Le Monde et de l'encyclopédie en ligne Wikipédia, vis-à- 
vis de laquelle la plupart des enseignants émettent d'ordinaire, non sans quelques motifs, de grandes réserves. Une manière de dire que la quasi-totalité des données qu'il présente sont assez généralement admises, et largement accessibles pour ceux qui veulent bien se donner la peine de les chercher. Professeur émérite d'économie de l'Université de Paris 7 - après avoir passé l'essentiel de sa carrière dans celle de Paris 8 -, il a en effet publié de vastes fresques sur l'histoire du capitalisme mondialisé ${ }^{1}$, et ses corollaires en termes d'inégalités et de dégradations écologiques. Bref, une certaine hauteur de vue ${ }^{2}$ devenue rare en ces temps de spécialisation croissante de la recherche, mais pourtant non moins indispensable pour percevoir la cohérence systémique d'une organisation économique et sociale devenue insoutenable dans tous les sens du terme. Malheureusement, le constat est désormais largement connu, et les «lanceurs d'alerte » écologistes au sujet du «basculement » du monde font désormais florès dans les librairies comme sur les ondes et jusque dans les cénacles institutionnels, sans pour autant suffire pour inverser le «cours des choses ». Encore faut-il séparer parmi eux ceux qui sont réellement sincères des " éco-tartuffes » surtout soucieux de s'ouvrir de nouveaux marchés par la promotion de la "croissance verte $»^{3}$, et que l'auteur s'applique ici à dénoncer avec insistance. Car la "crise » actuelle, s'emploie-t-il à montrer, est indissociablement environnementale, économique et sociale, et elle n'est rien d'autre que la marque de l'incurie du capitalisme dans ses principes mêmes, et cela en dépit de ses incessantes transformations adaptatives.

2 Telle est en effet la principale originalité de l'ouvrage de Michel Beaud, non pas tant la critique du capitalisme, que la mise en perspective historique qu'il en propose en revenant au début du millénaire dernier, où, d'après les impressionnantes séries établies sous la direction d'Angus Maddison ${ }^{4}$, l'activité économique stagnait sur l'ensemble de la planète, mais aussi et surtout, était également répartie. Ce n'est qu'avec les révolutions industrielles du 19e siècle que la croissance de la production a explosé, et avec elle les inégalités internationales. Autrement dit, c'est à ce moment qu'a commencé la fuite en avant qui a préparé le « basculement $»^{5}$ auquel nous devons aujourd'hui faire face.

3 L'auteur commence donc par décrire ce basculement, ce " pire des mondes » qui est à la fois déjà là et à venir si rien ne change, révélant ce faisant les enjeux qui lui paraissent les plus cruciaux. Il commence ainsi par égratigner sérieusement le mythe des «Trente Glorieuses » en rappelant que, dès cette période, les signes d'atteintes sérieuses à la nature étaient perceptibles (pluies acides, dégradation de la couche d'ozone) - et perçus -, sans être suffisamment pris au sérieux. Sans doute l'agenda politique très chargé de la Guerre froide, et en particulier la course à la croissance que celle-ci a impliqué, n'ont-elles pas favorisé de telles préoccupations qui apparaissaient secondaires. Sauf que sa fin n'a pas marqué de réel changement, sinon rhétorique. Dès 1992, le sommet de Rio a en effet apparemment marqué l'officialisation d'une volonté d'action collective contre le "changement climatique" et ses causes. Mais les oppositions faisant le jeu des intérêts industriels ainsi menacés ne se sont pas fait attendre. Les premiers à réagir ont même été certains scientifiques comme le rappelle Michel Beaud, dont 264, parmi lesquels plusieurs récipiendaires du Prix Nobel, signèrent la même année l'«appel de Heidelbeg" fustigeant la montée d'une « idéologie irrationnelle » menaçant la trinité du Progrès, de la Science et l'Industrie. Mais s'est surtout ouverte dès cette époque une tension entre pays riches et pays émergents, les seconds accusant les premiers de les empêcher par ce biais d'atteindre la 
prospérité. Aucun accord contraignant n'a alors pu être pris ni à Rio, ni au cours des sommets suivants, dont le dernier en date à Durban.

Michel Beaud revient ainsi en détail sur chacune de ces réunions et le contenu de leurs accords, ainsi que sur les positions parfois ambiguës mais souvent sans ambages de leurs participants - comme le président états-unien George Bush Junior affirmant que «le mode de vie américain n'était pas négociable ». Puis il détaille en parallèle les enjeux qui lui paraissent donc cruciaux et les développe avec pédagogie : non seulement les gaz à effet de serre et le changement climatique, mais aussi l'acidification des océans (pour laquelle le caractère géométrique de l'échelle des $\mathrm{pH}$ utilisée conventionnellement peut amener une dangereuse sous-estimation) et ses implications, destruction de la vie marine en tête, ainsi que les autres formes de pollution, comme les rejets de matières plastiques. Autre enjeu: la réduction progressive de la surface de terres arables et cultivées à l'échelle mondiale, conséquence de l'érosion et la désertification. Ces phénomènes découlent eux-mêmes de facteurs plus directement socio-politiques, dont les rachats de terres par des firmes ou États étrangers, que l'auteur compare à un nouveau mouvement des enclosures, ne sont qu'une des manifestations les plus éloquentes. L'essor des agro-carburants (que l'auteur continue étonnamment à nommer trompeusement «biocarburants») participe, quant à lui, de la déforestation, autre exemple de la dévastation de la nature décrite ici avec force chiffres.

5 La période actuelle est ainsi caractérisée, explique l'auteur, par trois «crises de reproduction » simultanées. Ce sont simultanément les conditions de reproduction de la Terre, de l'Humanité et du capitalisme qui sont remises en cause, ou, plus exactement, qui entrent en conflit. Or, celles-ci sont aujourd'hui hiérarchisées dans l'ordre inverse dans l'agenda politique (reproduction du capitalisme, puis de l'Humanité et enfin de la Terre), alors même que si la reproduction du capitalisme a besoin des deux autres, celle de l'Humanité a besoin de la Terre, mais cette dernière ne s'appuie pas sur les précédentes - au contraire, serait-on tenté de dire. Comment enrayer alors la spirale de "croissance destructrice »? L'auteur développe comme de bien entendu dans une dernière partie un certain nombre de propositions à cet égard sans pour autant détailler les conditions concrètes de leur mise en œuvre. Il prône ainsi quatre "ruptures» à réaliser sur le champ: rupture avec l' « ultralibéralisme », rupture avec l'existence de revenus et fortunes excessifs, rupture avec l'impunité dont jouissent de fait les décideurs économiques et politiques, et enfin rupture avec la spirale de consommation effrénée. Pour autant, il prend la peine de préciser certaines positions qui apparaîtront moins consensuelles à certains. En insistant sur le fait que la captation des ressources comme les pollutions sont en réalité le fait d'une minorité d'habitants de la planète dans les pays riches, il avance ainsi que le « problème » n'est pas selon lui dans la surpopulation, mais dans la surproduction et la surconsommation de cette minorité. Seul hic, qu'il n'est pas sans relever: ce "modèle» de vie gaspilleur attire bel et bien le reste du monde, dont une frange importante des populations asiatiques qui commencent à progressivement acquérir les moyens financiers d'y accéder. C'est bien la sortie de cet imaginaire qui allie bonheur et consommation dont il s'agit de s'affranchir, mais l'auteur ne livre guère de clés pour cela.

6 De même, Michel Beaud appelle-t-il à distinguer les mécanismes du marché de ceux du capitalisme, les premiers étant aussi créateurs de liens, pourvu qu'ils soient encadrés et 
réglementés, afin d'être mis au service de la population et contenu dans les limites de la reproduction de l'écosystème, contrairement à aujourd'hui. Il semble ainsi accorder une grande part de ses espoirs dans le renforcement des mécanismes et contraintes juridiques pour parvenir à instaurer et faire appliquer des normes plus contraignantes en matière d'environnement, d'inégalités de revenus et de patrimoines (en fixant à la fois des minima et maxima), et enfin pour mieux encadrer les institutions financières. Autrement dit, définir de véritables délits dans ces domaines pour punir réellement les responsables politiques et économiques, dirigeants des États et des Très grandes firmes, comme l'auteur les appelle, en insistant non sans raison sur leur poids écrasant dans la détermination des directions que prennent nos sociétés. Le rapport de force reste cependant plus que jamais en leur faveur, comme l'ont montré les derniers déroulements de la « résolution » d'une « crise globale» dont ces pompiers pyromanes s'avèrent bien habiles pour « faire payer la facture » aux autres.

7 L'auteur semble ainsi osciller entre une très grande lucidité quant à la gravité de la situation actuelle du monde, notamment sur le fait qu'on ne sortira pas du capitalisme " par décret », et entre, en même temps, un optimisme parfois naïf, peu attentif aux conflits entre intérêts divergents à court terme, notamment lorsqu'il espère le développement prochain de produits totalement dépourvus de pollutions et intégralement recyclés. Son souhait répété de voir émerger un leader charismatique pourra également en déconcerter plus d'un, lorsqu'il écrit par exemple : « il faudrait un personnage charismatique qui allie, dans la lutte pour la Terre et les humains, l'universalisme d'un Benjamin Franklin, l'obstination d'un Mandela, le courage d'un Allende, la ténacité d'un Gandhi, l'énergie d'un Churchill et la conviction d'un Jaurès. Mais nous avons eu Sarkozy, Clinton, Chirac, Bush, Blair, Berlusconi, et des dizaines d'autres du même acabit » (p. 235-236). Un statut de maître à penser qu'il ne faudrait donc pas non plus accorder à Michel Beaud, qui, comme tout un chacun, révèle ses propres contradictions, et ne propose pas non plus le livre-solution pour sortir de l'impasse où nous ne cessons de nous engouffrer collectivement. Il n'en offre pas moins une perspective à bien des égards originale qui, on peut au moins l'espérer, parviendra à convaincre quelques réticents supplémentaires des enjeux réels auxquels nous avons à faire face.

\section{NOTES}

1. Voir notamment son ouvrage "classique", cinq fois réédité et réactualisé : Histoire du capitalisme. 1500-2010, Paris, Seuil, 2010 [1981].

2. Pour s'en faire une idée, voir sa page personnelle : http://www.michelbeaud.com/ [Consultée le 30 décembre 2011].

3. Avant l'arrivée de ce slogan non dénué de contradictions, l'auteur cite à plusieurs reprises l'exemple de la firme Dupont, principal producteur des gaz CFC. Ses dirigeants ont activement œuvré contre leur interdiction jusqu'au jour où, leurs ingénieurs ayant développé des alternatives, ils ont soudainement radicalement changé leur fusil d'épaule, toujours dans le souci de garantir leurs profits.

4. Voir notamment L'économie mondiale, une perspective millénaire, Paris, éd.de l'OCDE, 2001. 
5. Expression désormais pratiquement usuelle et qui a donné son titre à deux précédents ouvrages de l'auteur (Le basculement du monde, Paris, La Découverte, 1997, et Journal du basculement du monde, Paris, La Découverte, 2001).

\section{AUTEUR}

IGOR MARTINACHE

Igor Martinache est Prag en SES à l'Université de Paris-Est-Créteil 